\title{
Electro-Optical Properties of II-VI Superlattices
}

\author{
P. SChILlaK ${ }^{a, *}$ AND G. CzAJKOWSKI ${ }^{a, b}$ \\ ${ }^{a}$ University of Technology and Life Sciences \\ Kaliskiego 7, 85-796 Bydgoszcz, Poland \\ ${ }^{b}$ Scuola Normale Superiore \\ Piazza dei Cavalieri 7, 56126 Pisa, Italy
}

\begin{abstract}
We show how to compute electro-optical spectra of semiconductor superlattices in the region of interband electronic transitions. The method uses the microscopic calculation of eigenvalues and eigenfunctions and the macroscopic real density matrix approach to compute the electromagnetic fields and susceptibilities. The electron-hole screened Coulomb potential is adapted and the valence band structure is taken into account in the cylindrical approximation, thus separating light- and heavy-hole motions. We calculate the electro-optical functions, including the optical Stokes parameters and ellipsometric functions for the case of oblique incidence. Results are given for $\mathrm{Zn}_{1-x} \mathrm{Cd}_{x} \mathrm{Se} / \mathrm{ZnSe}$ superlattices and a good agreement with experiments is obtained.
\end{abstract}

PACS numbers: 71.35.Cc, 73.21.Cd, 74.25.Gz, 78.20.Jq, 78.67.-n

\section{Density matrix formulation for superlattices}

Below we compute the electro-optical functions of superlattices (SLs) when a static electric field $\boldsymbol{F}$ is applied in the growth direction, which is identified as the $z$-direction. We consider the low barriers limit when the electron and hole motion in the growth direction is determined by the superlattice potential and is replaced by an effective mass motion with appropriate effective masses obtained from the miniband dispersion relations [1,2]. From the miniband dispersion we obtain the bands $C \nu=1,2, \ldots$ in the conduction band and $H \mu=1,2, \ldots, L \mu=$ $1,2, \ldots$ in the valence band. By the SL selection rule we consider the (dipole allowed) transitions $H, L \nu \rightarrow C \nu$ and denote them by $H, L \nu$. To obtain the optical response we apply the real density matrix approach [3-5] taking into account both the Coulomb interaction between the electron and the hole, and a coherence between the electron-hole pair and the radiation field. In this approach the linear response is described by a set of coupled equations (constitutive equations, CEs) for two-point correlation functions $\mathcal{Y}_{12 H \nu}$ and $\mathcal{Y}_{12 L \nu}$ for the $\nu$-th heavy-hole exciton

*corresponding author; e-mail: psc@utp.edu.pl 
$(H \nu)$ and the $\nu$-th light-hole exciton $(L \nu)$, and a Maxwellian field equation; $\nu$ labels the respective couple of minibands. The CEs have a form of Schrödinger's equation with a source term, which reflects the light-matter interaction. The operator $H_{e h \nu}$, which occurs in the above equations, is the SL exciton effective mass Hamiltonian for the $\nu$-th couple of bands, containing kinetic energy terms, screened Coulomb interaction, and the term $e F\left(z_{\mathrm{e}}-z_{\mathrm{h}}\right)$ describing the carriers' interaction with the applied electric field. The carriers effective masses, occurring in the Hamiltonian, can be computed by the Kronig-Penney model (see, for example, [6]). The functions $\mathcal{Y}_{H \nu}, \mathcal{Y}_{L \nu}$ give the total polarization of our effective anisotropic medium

$$
\mathcal{P}(\boldsymbol{R}, t)=2 \sum_{\nu} \int \mathrm{d}^{3} r\left[\boldsymbol{M}_{H \nu}^{*}(\boldsymbol{r}) \mathcal{Y}_{H \nu}(\boldsymbol{r}, \boldsymbol{R}, t)+\boldsymbol{M}_{L \nu}^{*}(\boldsymbol{r}) \mathcal{Y}_{L \nu}(\boldsymbol{r}, \boldsymbol{R}, t)\right],
$$

where $\boldsymbol{M}_{\nu}$ is the dipole density appropriate to the transition $H, L \nu \rightarrow C \nu$. The above equation with the constitutive equations connect the polarization with the electric field of the propagating wave. Both polarization and electromagnetic field must obey Maxwell's equations, which must be solved to obtain the propagation modes.

\section{SL excitonic susceptibility tensor and optical functions}

We want to compute the linear response to an arbitrary polarized wave propagating in a direction $\boldsymbol{k}$ :

$$
\mathcal{E}(\boldsymbol{R}, t)=\boldsymbol{E} \mathrm{e}^{\mathrm{i} \boldsymbol{k} \boldsymbol{R}-\mathrm{i} \omega t} .
$$

The electric field $\boldsymbol{E}$ is obtained in a self-consistent way from CEs and the Maxwell equation, where the polarization (1) acts as a source. In the first step we computed the functions $\mathcal{Y}_{\nu}$ by means of eigenfunctions $\varphi$ of the SL Hamiltonian. The calculation of these eigenfunctions, due to the dimensionality of the problem (6-dimensional configuration space) and of the Coulomb potential, is a complicated problem. Here we only sketch the way of solution: the details will be given elsewhere [7]. By the strong SLs anisotropy we can separate the fast in-plane carriers movement, expanding the eigenfunction in series

$$
\varphi(Z, \boldsymbol{r})=\sum_{n, m=0}^{\infty} f_{n m}(Z, z) \psi_{n m}(\rho, \phi)
$$

$\psi_{n m}(\rho, \phi)$ being the known eigenfunctions of the quantum two-dimensional Kepler problem, and $f_{n m}(Z, z)$ unknown functions to be established; the in-plane center of mass movement has been separated by the factor $\exp \left(\mathrm{i} \boldsymbol{K}_{\|} \boldsymbol{R}_{\|}\right)$. The functions $f_{n m}$ obey an infinite system of equations

$$
\begin{aligned}
& \left(\varepsilon_{n_{1} m_{1}}-\frac{\mu_{\|}}{\mu_{z}} \frac{\partial^{2}}{\partial z^{2}}+\frac{\mu_{\|}}{M_{\|}} K_{\|}^{2}-\frac{\mu_{\|}}{M_{z}} \frac{\partial^{2}}{\partial Z^{2}}+e F z\right) f_{n_{1}, m_{1}}(Z, z) \\
& +\sum_{n_{2} m_{2}}\left(v_{n_{1} m_{1} n_{2} m_{2}}-V_{n_{1} m_{1} n_{2} m_{2}}\right) f_{n_{2} m_{2}}(Z, z)=\varepsilon f_{n_{1} m_{1}}(Z, z),
\end{aligned}
$$


$\varepsilon_{n m}$ being the eigenvalues of the 2-dimensional (2D) Kepler problem, and $v, V$ the potential matrix elements

$$
\begin{aligned}
& v_{n_{1} m_{1} n_{2} m_{2}}=\left\langle\psi_{n_{1} m_{1}} \mid \frac{2}{\rho} \psi_{n_{2} m_{2}}\right\rangle, \\
& V_{n_{1} m_{1} n_{2} m_{2}}=\left\langle\psi_{n_{1} m_{1}}\left|\frac{2}{\sqrt{\rho^{2}+z^{2}}}\right| \psi_{n_{2} m_{2}}\right\rangle .
\end{aligned}
$$

In the next step we transform the system of Eqs. (4) into a system of algebraic equations, taking into account the functions $f_{00}, f_{10}, f_{20} \ldots$ As the results we obtain the eigenenergies and eigenfunctions which are used to solve the constitutive equations.

\section{Results}

Using the eigenfunctions and eigenvalues we computed the polarization and the electric field of the electromagnetic wave propagating in the SL. Having this, we could determine the electro-optical functions for a superlattice: we have chosen the $\mathrm{Zn}_{0.8} \mathrm{Cd}_{0.2} \mathrm{Se} / \mathrm{ZnSe} \mathrm{SL}$ of the thickness of $50 \mathrm{~nm}$. As an example, the numerical results for the real and imaginary part of the susceptibility and for two values of the applied electric field are displayed in Fig. 1. For the case without the field we
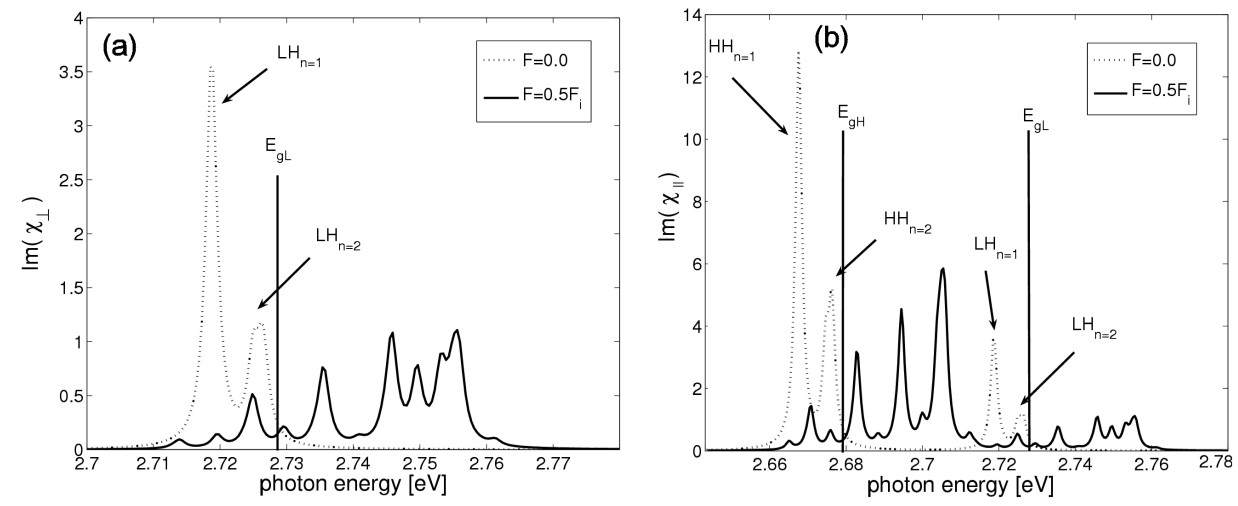

Fig. 1. Imaginary part of the electro-susceptibility tensor for a $\mathrm{Zn}_{0.8} \mathrm{Cd}_{0.2} \mathrm{Se} / \mathrm{ZnSe} \mathrm{SL}$ : (a) in the direction perpendicular to the layers, (b) in the direction parallel to the SL layers, for two values of the applied electric field $F=0$ (dotted line) and $F=0.5 F_{\mathrm{I}}$ (solid line).

observe peaks corresponding to the $s$-states $H n=1,2$ and $L n=1,2$ (depending on the polarization of the incoming wave). When the constant field is applied, the Franz-Keldysh oscillations occur for the energies above the respective gap energies. Their amplitudes and periodicity depend on the field strength and increase with the increasing field. Having the susceptibility tensor, we can determine the dielectric tensor, $\underline{\underline{\epsilon}}=\epsilon_{b}+\underline{\underline{\chi}}$ and hence all the optical properties of a superlattice. For the 


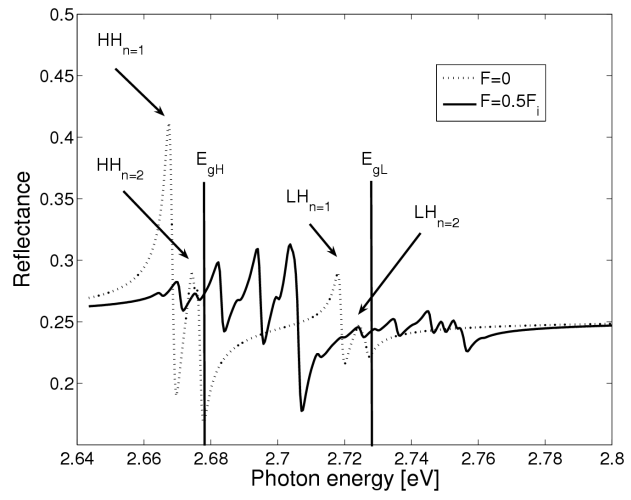

Fig. 2. Normal incidence reflectance for a $\mathrm{Zn}_{0.8} \mathrm{Cd}_{0.2} \mathrm{Se} / \mathrm{ZnSe} \mathrm{SL}$ of the thickness of $50 \mathrm{~nm}$.

purpose of illustration we calculated the SL reflectance for two values of the applied field. Again we observe the Franz-Keldysh oscillations above the respective gap energies (Fig. 2). We were also able to compute the optical Stokes parameters and ellipsometric parameters $\Psi$ and $\Delta$ (not shown).

\section{Conclusions}

For the first time we present the full numerical solution of the eigenvalue problem of the SL exciton interacting with an applied static electric field. Having this solution we computed oblique incidence electro-optical functions for a $\mathrm{Zn}_{1-x} \mathrm{Cd}_{x} \mathrm{Se} / \mathrm{ZnSe} \mathrm{SL}$. Our results are in qualitative agreement with the experimental results of Ref. [8].

\section{References}

[1] G. Bastard, Wave Mechanics Applied to Semiconductor Heterostructures, Les Editions de Physique, Paris 1989.

[2] M.F. Pereira Jr., I. Galbraith, S.W. Koch, G. Duggan, Phys. Rev. B 42, 7084 (1990).

[3] A. Stahl, I. Balslev, Electrodynamics of the Semiconductor Band Edge, Springer-Verlag, Berlin 1987.

[4] G. Czajkowski, M. Dressler, F. Bassani, Phys. Rev. B 55, 5243 (1997).

[5] G. Czajkowski, F. Bassani, L. Silvestri, Rivista del Nuovo Cimento C 26, 1 (2003).

[6] P. Schillak, G. Czajkowski, Phys. Status Solidi B 244, 1627 (2007).

[7] P. Schillak, G. Czajkowski, to be published.

[8] Z.P. Guan, T. Kobayashi, Solid State Commun. 111, 311 (1999). 